\title{
Brockenbrough-Braunwald-Morrow Sign: An Evaluative Hemodynamic Maneuver for Left Ventricular Outflow Tract Obstruction
}

\author{
Glenmore Lasam
}

\begin{abstract}
A case of a 77-year-old woman with a history of hypertrophic cardiomyopathy (HCM) presented with intermittent episodes of exertional dyspnea and chest discomfort. Her coronary angiogram revealed normal coronary arteries but with hypertrophic obstructive cardiomyopathy with an increasing left ventricular-aortic gradient on isoproterenol provocation. Likewise, an intensified gradient was observed after a premature ventricular contraction (PVC) that is distinguished as the Brockenbrough-Braunwald-Morrow sign substantiating confirmation of left ventricular outflow tract (LVOT) obstruction.
\end{abstract}

Keywords: Brockenbrough-Braunwald-Morrow sign; Hemodynamic maneuver; Left ventricular outflow tract obstruction; Arterial pulse pressure; Left ventricular systolic pressure; Premature ventricular contraction

\section{Introduction}

Hypertrophic cardiomyopathy (HCM) presents with left ventricular outflow tract (LVOT) obstruction culminating into syncope, angina and heart failure symptoms. An evaluative hemodynamic maneuver, Brockenbrough-Braunwald-Morrow Sign, can provide a hint to the existence and degree of LVOT obstruction.

\section{Case Report}

A 77-year-old woman with a history of HCM, hypertension, and hyperlipidemia presented to her cardiologist because of intermittent episodes of exertional dyspnea and chest discomfort. She has been on anti-ischemic treatment but did not improve her symptoms. She was eventually scheduled for an elective cardiac catheterization which showed normal coronary arteries, mild pulmonary hypertension, elevated pulmo-

Manuscript submitted December 31, 2017, accepted February 8, 2018

Morristown Medical Center, Morristown, NJ 07960, USA.

Email: glenmore_md@yahoo.com

doi: https://doi.org/10.14740/cr676w nary capillary wedge and left ventricular end diastolic pressures, and hypertrophic obstructive cardiomyopathy (HOCM) with an increasing left ventricular-aortic gradient on isoproterenol challenge. A left ventricular and aortic pressure gradient was absent at rest (Fig. 1), but was recognized during isoproterenol administration (Fig. 2). Also, an intensified gradient was observed after a premature ventricular beat (Fig. 2) that is characterized as the Brockenbrough-Braunwald-Morrow sign establishing evidence of LVOT obstruction.

Based on the results of coronary angiography with hemodynamic evaluation, the patient was advised to undergo either septal alcohol ablation or surgical myomectomy to abate her symptoms attributed from the HOCM.

\section{Discussion}

Brockenbrough-Braunwald-Morrow sign has been conceptualized when a paradoxical decline in arterial pulse pressure and a concomitant rise in left ventricular systolic pressure were noted post premature ventricular contraction beat [1]. A compensatory pause ensues following a premature ventricular contraction (PVC) which increases the filling time during diastole, subsequently, expands the diastolic volume.

The Frank-Starling mechanism posits that the stroke volume increases as a result of an increase in end diastolic volume when all factors remain constant [2]. Consequently, the expanded end diastolic volume increases cardiac muscle stretch resulting in an increase in myocardial contractility. Extracellular calcium enters the cardiomyocytes during extrasystole, accumulates in the sarcoplasmic reticulum, and significantly contributes during post extrasystolic contraction [3].

The augmented inotropic response following a PVC aggravates obstruction of the LVOT precipitating a drop in arterial pulse pressure and elevation of left ventricular systolic pressure [4]. Hemodynamic maneuver in the catheterization laboratory utilizes the isoproterenol to document the worsening of the LVOT obstruction in patients with HCM which was evident with this patient. Nethalide, a beta blocker has been used to reverse the effect of isoproterenol which evolutionized the use of beta blockers as the initial treatment of choice in HCM especially those patients with angina [5-7]. Also, an echocardiographic case of intermittent LVOT obstruction had been reported following a premature atrial contraction described as systolic anterior motion of the anterior mitral valve leaflet with prolonged systolic septal contact, 


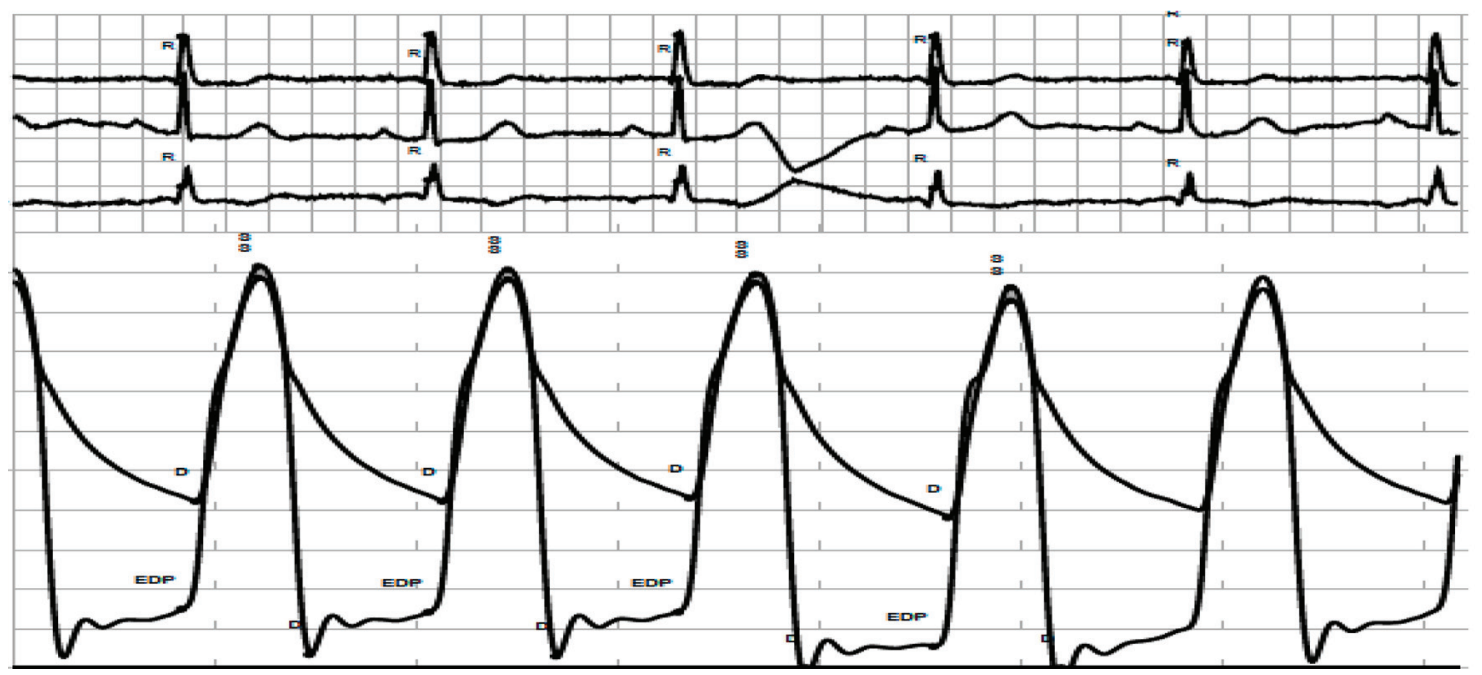

Figure 1. Concurrent measurement at rest of left ventricular and aortic pressures on a gradation of 0 to $200 \mathrm{~mm} \mathrm{Hg}$ demonstrating an absence of left ventricular outflow tract gradient.

dynamic LVOT obstruction, and significant mitral regurgitation which were not evident during the preceding contraction [8].

Brockenbrough-Braunwald-Morrow sign is a beneficial catheterization laboratory maneuver that can be utilized in patients with substantial symptoms and has an exertional gradient not seen at rest [9]. HOCM patients with no documented gradient at rest are around $75 \%$, and the maneuver can be employed to note the severity of dynamic LVOT obstruction in perioperative alcohol septal ablation [10] as well as to confirm the abolition of subaortic obstruction post procedure [11].

\section{Conclusion}

The utilization of the hemodynamic maneuver, Brocken-
brough-Braunwald-Morrow sign, is an essential evaluative modality in HCM patients with dynamic LVOT obstruction.

\section{Conflict of Interest}

The author declares that he has no conflict of interest.

\section{References}

1. Brockenbrough EC, Braunwald E, Morrow AG. A hemodynamic technique or the detection of hypertrophic subaortic stenosis. Circulation. 1961;23:189-194.

2. Widmaier EP, Hershel R, Strang KT. Vander's Human

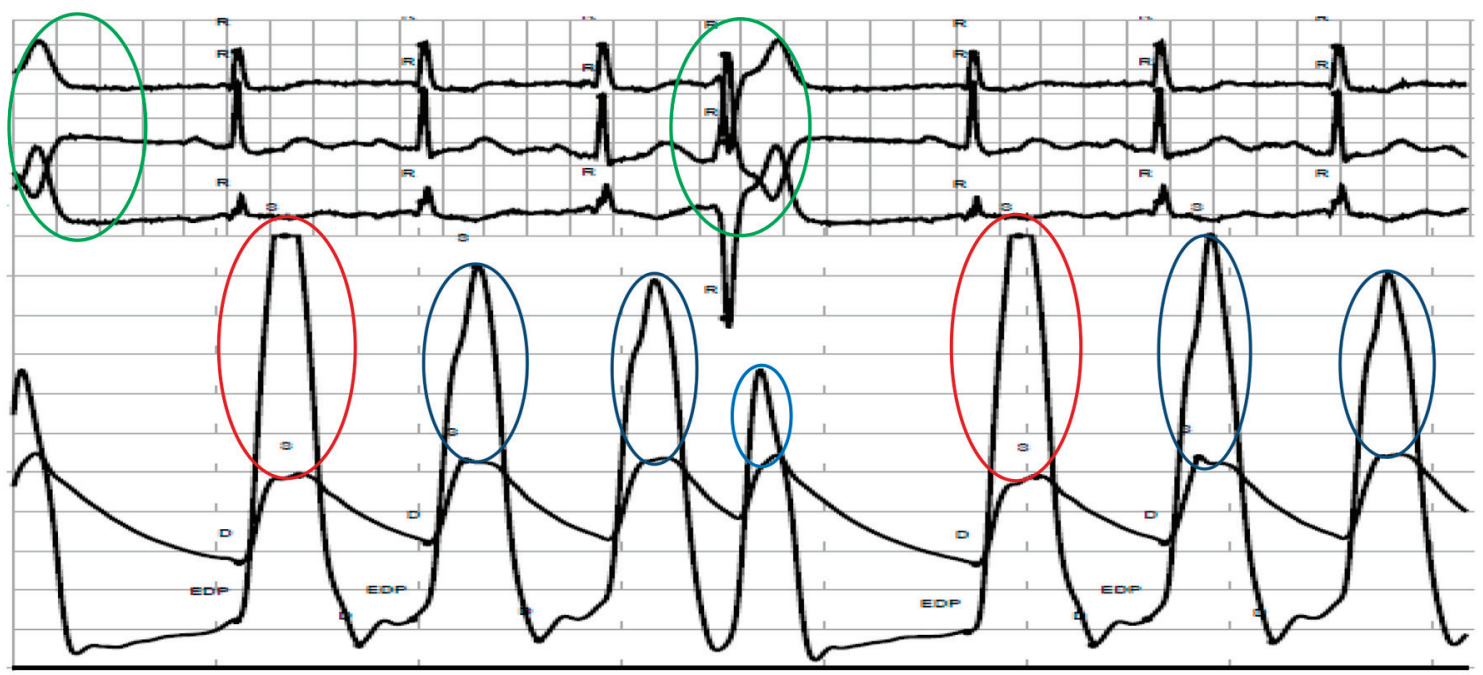

Figure 2. Concurrent measurement of left ventricular and aortic pressures after isoproterenol administration exhibiting evidence of $120 \mathrm{~mm} \mathrm{Hg}$ gradient (blue oval at lower panel). Also, an intensified left ventricular-aortic gradient (red oval at lower panel) was noted after premature ventricular contraction (green oval at upper panel). 
Physiology: The Mechanisms of Body Function (14th ed.). New York, NY: McGraw-Hill Education. 2016. ISBN 978-1-259-29409-9.

3. Marengo FD, Marquez MT, Bonazzola P, Ponce-Hornos JE. The heart extrasystole: an energetic approach. Am J Physiol. 1999;276(1 Pt 2):H309-316.

4. Pollock SG. Images in clinical medicine. Pressure tracings in obstructive cardiomyopathy. N Engl J Med. 1994;331(4):238.

5. Harrison DC, Braunwald E, Glick G, Mason DT, Chidsey CA, Ross J, Jr. Effects of beta adrenergic blockade on the circulation with particular reference to observations in patients with hypertrophic subaortic stenosis. Circulation. 1964;29:84-98.

6. Cherian G, Brockington IF, Shah PM, Oakley CM, Goodwin JF. Beta-adrenergic blockade in hypertrophic ob- structive cardiomyopathy. Br Med J. 1966;1(5492):895898.

7. Wigle ED, Rakowski H, Kimball BP, Williams WG. Hypertrophic cardiomyopathy. Clinical spectrum and treatment. Circulation. 1995;92(7):1680-1692.

8. Adawi S, Asmer I, Merdler A, Shiran A. Echocardiographic Brockenbrough-Braunwald-Morrow sign. Eur J Echocardiogr. 2011;12(3):E12.

9. Trevino AR, Buergler J. The Brockenbrough-Braunwald-Morrow sign. Methodist Debakey Cardiovasc J. 2014;10(1):34-37.

10. Maron BJ. Hypertrophic cardiomyopathy. Lancet. 1997;350(9071):127-133.

11. Soon CY, Buergler JM. Alcohol septal ablation and the Brockenbrough-Braunwald phenomenon. Catheter Cardiovasc Interv. 2008;72(7):1016-1024. 